\title{
PCPH expression is an early event in the development of testicular germ cell tumors
}

\author{
JAVIER REGADERA ${ }^{1}$, MARÍA JOSÉ BLÁNQUEZ ${ }^{2}$, PILAR GONZÁLEZ-PERAMATO ${ }^{3}$, \\ MANUEL NISTAL ${ }^{1,4}$, JENNIFER C. MILLER ${ }^{5}$, OSCAR M. TIRADO ${ }^{5}$ and VICENTE NOTARIO ${ }^{5}$ \\ ${ }^{1}$ Department of Anatomy, Histology and Neurosciences, School of Medicine, Autónoma University; \\ ${ }^{2}$ Department of Anatomy, Veterinary School, Complutense University; ${ }^{3}$ Department of Pathology, \\ Guadalajara General Hospital, University of Alcalá, Alcalá de Henares; ${ }^{4}$ Department of Pathology, \\ La Paz Hospital, Madrid, Spain; ${ }^{5}$ Laboratory of Experimental Carcinogenesis, Department of \\ Radiation Medicine, Georgetown University Medical Center, Washington, DC, USA
}

Received September 19, 2005; Accepted October 24, 2005

\begin{abstract}
Testicular germ cell tumors (TGCTs) include various malignancies with distinct pathologies that share a common precursor lesion (intratubular germ cell neoplasia, unclassified, ITGCNU, or carcinoma in situ, CIS). TGCTs, as a whole, represent a highly curable tumor paradigm, with high sensitivity to radiotherapy and, especially, to cisplatinbased chemotherapy. However, a percentage of cases display therapeutic resistance, and the molecular mechanisms underlying such resistant phenotype remain to be elucidated. We put forward the notion that expression of oncogenic forms of the $P C P H$ gene, which are known to confer resistance to radiation and chemotherapeutic drugs, including cisplatin, may be expressed in TGCTs, and thus contribute to the development of therapeutic resistance. To begin testing this concept, we studied PCPH expression in human TGCT cell lines and in 54 solid tumors by RT-PCR, Western immunoblot and immunohistochemistry. The results demonstrated that: i) PCPH is expressed in TGCT cell lines and tumors, including CIS; ii) its expression levels vary among different TGCT pathologies, being generally higher in well differentiated regions and lower in areas of predominant proliferation; iii) PCPH expression is substantially increased in tumors relative to matched normal testicular tissue; iv) tumor samples express PCPH polypeptides of low molecular mass, consistent with the known size of the PCPH oncoprotein, that are either absent from, or markedly reduced in, matched normal tissue.
\end{abstract}

Correspondence to: Dr V. Notario, Laboratory of Experimental Carcinogenesis, Department of Radiation Medicine, Georgetown University Medical Center, Research Building, Room E215, Washington, DC 20057, USA

E-mail: notariov@georgetown.edu

Key words: germ cell tumors, oncogenes, $\mathrm{PCPH}$, testis, testicular tumors
Collectively, these results positively identify PCPH as a good early molecular marker for testicular neoplasms, and strongly indicate that immunodetection of truncated PCPH polypeptides may be a useful diagnostic tool for TGCT.

\section{Introduction}

$P C P H$ is a gene involved in the regulation of cell proliferation, as evidenced by its initial identification as an oncogene, formerly termed $c p h$ (1), and of the cellular response to stress $(2,3)$. Indeed, promotion of resistance to stress stimuli such as nutritional starvation, ionizing radiation or chemotherapeutic agents contributes to the neoplastic transformation activity of the $P C P H$ oncogene (2). The survival-promoting function of the PCPH oncoprotein is mediated by its ability to antagonize proapoptotic signaling pathways activated by exposure to ionizing radiation (4) or chemotherapeutic drugs such as cisplatin (Tirado et al, Proc Am Assoc Cancer Res 45: 1158, abs. 5017, 2004). Because recent analyses of human and animal solid tumors and cell lines indicated that deregulated expression of the PCPH protein is frequently associated with, and thus may participate in, tumor progression (5-8), it is likely that tumors expressing oncogenic forms of $\mathrm{PCPH}$ may be more resistant to radiotherapy and/or chemotherapy.

Testicular germ cell tumors (TGCT) represent approximately $95 \%$ of all testicular neoplasias, and are the fourth most frequent malignancy in young males. The incidence of TGCT has increased in recent years due to improvements of early detection in patients with a high risk of developing testicular tumors, primarily those with cryptorchidism and infertility (9-11). Seminomas are the most frequent testicular tumors in mid-aged patients, whereas non-seminomatous germinal tumors (embryonal carcinoma, endodermal sinus tumor) and teratomas have a greater incidence in children and young patients (10-12). Most TGCT forms are believed to derive from a common precursor of neoplastic intratubular germ cells (intratubular germ cell neoplasia, unclassified, ITGCNU, also called testicular carcinoma in situ, CIS) (13). The clinical behavior and evolution of TGCTs, as well as their response to therapy, vary considerably among morphological 
types, with seminomatous tumors being typically sensitive to radiation and chemotherapeutic drugs such as cisplatin, whereas non-seminomatous tumors are more resistant, especially to ionizing radiation (14). Prognostic factors, such as presence of metastasis and serum levels of specific tumor markers $(10,12,14)$, are also considered for treatment protocol planning. The best results are typically reported in the treatment of testicular cancers at stages I and II $(15,16)$. Recently, new chemotherapy protocols for TGCTs are yielding good results, especially with the use of cisplatin to treat non-seminomatous tumors (17). However, the molecular basis for the general sensitivity of TGCT to chemotherapy and/or radiotherapy is not fully understood. Various reports indicate that it is independent from the status of the tumor suppressor protein Tp53 $(14,18,19)$, the expression levels of anti/pro-apoptotic proteins such as Bcl-2 or Bax $(14,18,19)$, or FAS gene mutations $(20,21)$. Recent results linking resistance to treatment with Hsp27 (22) or SRPK1 (23) expression levels need further, independent confirmation.

Although testicular malignancies constitute an excellent example of highly curable tumors (24), up to $20 \%$ of patients develop resistance, manifested by either an incomplete response or relapse (23). Moreover, up to $30-40 \%$ of mature teratoma patients have been reported to have metastasis remnants after chemotherapy (10). Because $P C P H$ mRNA has been detected in total extracts of testicular tissue from Syrian hamsters (25), mice (26) and humans (27), it seemed possible that the presence of oncogenic forms of PCPH in TGCT or the deregulation of its expression at some stage during tumor onset or progression may contribute to the development of resistance of TGCT to radio- and/or chemotherapeutic treatment. To begin investigating such a possibility, we examined the frequency and spectrum of possible alterations in PCPH expression in TGCT cell lines and tumors by means of immunohistochemical and Western immunoblot approaches. The results indicated that PCPH is indeed expressed in TGCT cell lines and in tumors at all progression stages, including CIS, whereas in normal testicular tissue low levels of PCPH expression are restricted to Leydig and Sertoli cells with essentially undetectable expression in germ line cells, including the spermatogonia. Our data also showed that TCGT express PCPH polypeptides of low molecular mass that are either not expressed or expressed at markedly lower levels in matched normal tissue. These findings identify PCPH as a good marker for TGCT and suggest that detection of small PCPH polypeptides may be a useful tool for early diagnosis of testicular neoplasias.

\section{Materials and methods}

Cells, culture conditions and treatments. Human embryonal carcinoma cell lines, NT2/D1 (CRL-1973) and NCCIT (CRL-2073), were purchased from the American Type Culture Collection (ATCC, Manassas, VA) and cultured as recommended by the ATCC in their DMEM or RPMI media supplemented with $10 \%$ fetal bovine serum and antibiotics (penicillin and streptomycin) at $37^{\circ} \mathrm{C}$ in a humidified atmosphere of $5 \% \mathrm{CO}_{2}$ and $95 \%$ air. For differentiation experiments, the media of exponentially growing cell cultures were supplemented with retinoic acid (RA), prepared as a stock solution in dimethyl sulfoxide (DMSO), to a final concentration of $10 \mu \mathrm{M}$ for various time periods. Control cultures were exposed to an identical volume of DMSO. After RA treatment, cells were examined under the microscope to detect any possible evidence of differentiation and then lysed to generate total extracts for RNA and protein analyses.

Pathological specimens. A total of 54 testicular tumors diagnosed at the Department of Pathology of the La Paz University Hospital of Madrid, Spain, were included in this study. Tumor samples derived from patients between the ages of 7 and 64 included 21 cases of pure seminoma and 33 cases of non-seminomatous tumors. The latter samples included 5 cases of embryonal carcinoma, 4 cases of endodermal sinus tumor, 4 mature teratoma and 7 cases of immature teratoma (all of them pathologically uniform), as well as 10 cases of mixed tumors. Whenever possible, samples were divided into two approximately equal portions, one of which was processed immediately for immunohistochemistry while the other was snap-frozen in liquid nitrogen and stored at $-80^{\circ} \mathrm{C}$ for Western immunoblot analysis.

Preparation of total cell and tumor extracts. Exponentially growing cells were washed twice in cold phosphate-buffered saline (PBS), lysed in RIPA buffer containing protease inhibitors $(1 \mathrm{mM}$ PMSF, $10 \mathrm{mg} / \mathrm{ml}$ aprotinin and $10 \mathrm{mg} / \mathrm{ml}$ leupeptin) and sonicated for $5 \mathrm{sec}$, and the lysates were centrifuged at $13,000 \mathrm{~g}$ at $4^{\circ} \mathrm{C}$ for $30 \mathrm{~min}$. Supernatants were aliquoted and stored at $-80^{\circ} \mathrm{C}$ until use. Solid frozen tumors were pulverized while in liquid nitrogen, suspended in lysis buffer and disrupted with the help of a Dounce homogenizer. The homogenate was centrifuged in a refrigerated microfuge, and the supernatant was used similarly to the cell extracts for RNA and protein analyses. The protein content in the extracts was determined using the BCA protein assay system (Pierce, Rockford, IL).

Immunohistochemistry. Surgical specimens were fixed in a $10 \%$ formalin solution at neutral $\mathrm{pH}$ for $24-48 \mathrm{~h}$, and embedded in paraffin. Serial sections $(6 \mu \mathrm{M}$ thick $)$ were processed essentially as described $(7,8)$ using an anti-PCPH antiserum (4) at a dilution of 1:4000. In all cases, slides were counterstained with hematoxylin. Serial sections of the same samples processed identically but not incubated with the primary antibody were used as negative controls. Positive controls included other human normal and tumor tissues known to express PCPH (5-8).

Western blot analysis. Proteins $(30 \mu \mathrm{g})$ in cell extracts were resolved by $10 \%$ SDS-polyacrylamide gel electrophoresis and transferred to nitrocellulose membranes. After blocking with 5\% non-fat dry milk in PBS containing $0.2 \%$ Tween-20, membranes were incubated at $4{ }^{\circ} \mathrm{C}$ overnight with the same anti-PCPH antibody as above, at a 1:10000 dilution. After extensive washing, membranes were incubated for $1 \mathrm{~h}$ with a horseradish peroxidase (HRP)-conjugated secondary antibody (1:2000). After washing, the peroxidase activity was analyzed using the ECL chemiluminescence substrate system (Amersham Biosciences, Piscataway, NJ). Blots were stripped and reprobed using an anti-human $B$-actin monoclonal antibody (Amersham) to control for equal sample loading. 
RNA analysis by $R T-P C R$. RNA was extracted using TRIzol reagent (Invitrogen/Life Technologies, Carlsbad, CA). Total RNA $(3 \mu \mathrm{g})$ was reverse transcribed using $200 \mathrm{U}$ of Superscript II RNase H-reverse transcriptase (Invitrogen) in a $20 \mu \mathrm{l}$ reaction volume, in the presence of $25 \mu \mathrm{g} / \mathrm{ml}$ Oligo(dT), first strand buffer ( $50 \mathrm{mM}$ Tris- $\mathrm{HCl}, 75 \mathrm{mM} \mathrm{KCl,} 3 \mathrm{mM} \mathrm{MgCl}$ ), $10 \mathrm{mM}$ dithiothreitol and $10 \mathrm{mM}$ each of dATP, dGTP, dCTP and dTTP. The mixture of RNA and Oligo(dT) was heated at $70^{\circ} \mathrm{C}$ for $10 \mathrm{~min}$ and cooled to $4^{\circ} \mathrm{C}$; then, all other reagents were added and reverse transcription was performed at $42^{\circ} \mathrm{C}$ for $50 \mathrm{~min}$. PCR products were resolved on $1.5 \%$ agarose gels. All PCR primers were designed using the Oligo 6.0 software from National Biosciences (Plymouth, MN), using the GeneBank published sequences as templates. A 1448-bp PCPH fragment was amplified using GGTGTGCGAGCAGG ATTG (upper) and GGAGATGCCCAGAGACTG (lower) as primers, and the primer set CGGGACCTGACTGACTAC CTC (upper) and CTTCATTGTGCTGGGTGC (lower) was used to amplify human actin, as standard for semi-quantitative determinations. For each set of cDNA-specific primers, the number of cycles was adjusted so that the reaction end-points fell within the exponential phase of product amplification $(28,29)$, thus providing a semi-quantitative estimate of relative mRNA abundance.

\section{Results}

TGCT cell lines express multiple PCPH polypeptide forms. We first investigated whether PCPH was expressed in TGCT cell lines and, if so, which polypeptide forms could be detected. Two cell lines were included in these studies: NCCIT, cells expressing mutant $\mathrm{Tp} 53$ that have undifferentiated properties equivalent to a stage intermediate between seminoma and embryonal carcinoma; and NT2/D1, malignant pluripotent embryonal carcinoma cells expressing wild-type Tp53. Total protein extracts prepared from exponentially growing cultures of both cell lines were analyzed by Western immunoblot using anti-PCPH antiserum. The results showed that both cell lines expressed several PCPH-immunorelated polypeptides with apparent molecular masses in the range of about $20-90 \mathrm{kDa}$ (Fig. 1), a pattern similar to that described for a variety of human cell lines derived from other tumor types (5). The low molecular-size PCPH polypeptide forms appeared to be much less abundant in NT2/D1 than in NCCIT cells. Interestingly, this pattern of expression remained invariable in total extracts of the same cell lines after extended treatment (up to 2 weeks) with all-trans retinoic acid (Fig. 1A, RA lane), a known inducer of differentiation of these cells to neuroectodermal-like phenotypes $(30,31)$, relative to untreated controls. The pattern and overall intensity of the various PCPH polypeptides were essentially the same in total extracts of cells treated for the same period of time with dimethyl sulfoxide (Fig. 1A, D lane), which was used to solubilize retinoic acid. These data indicated that, at least in culture, $\mathrm{PCPH}$ expression was not related to the differentiation state of TGCT cells. We then performed an immunohistochemical pilot study including a limited number of normal and neoplastic testicular samples. The results indicated that, in normal testicular tissue, low levels of PCPH expression were detected in Leydig and Sertoli cells, whereas PCPH expression remained at trace levels or was essentially
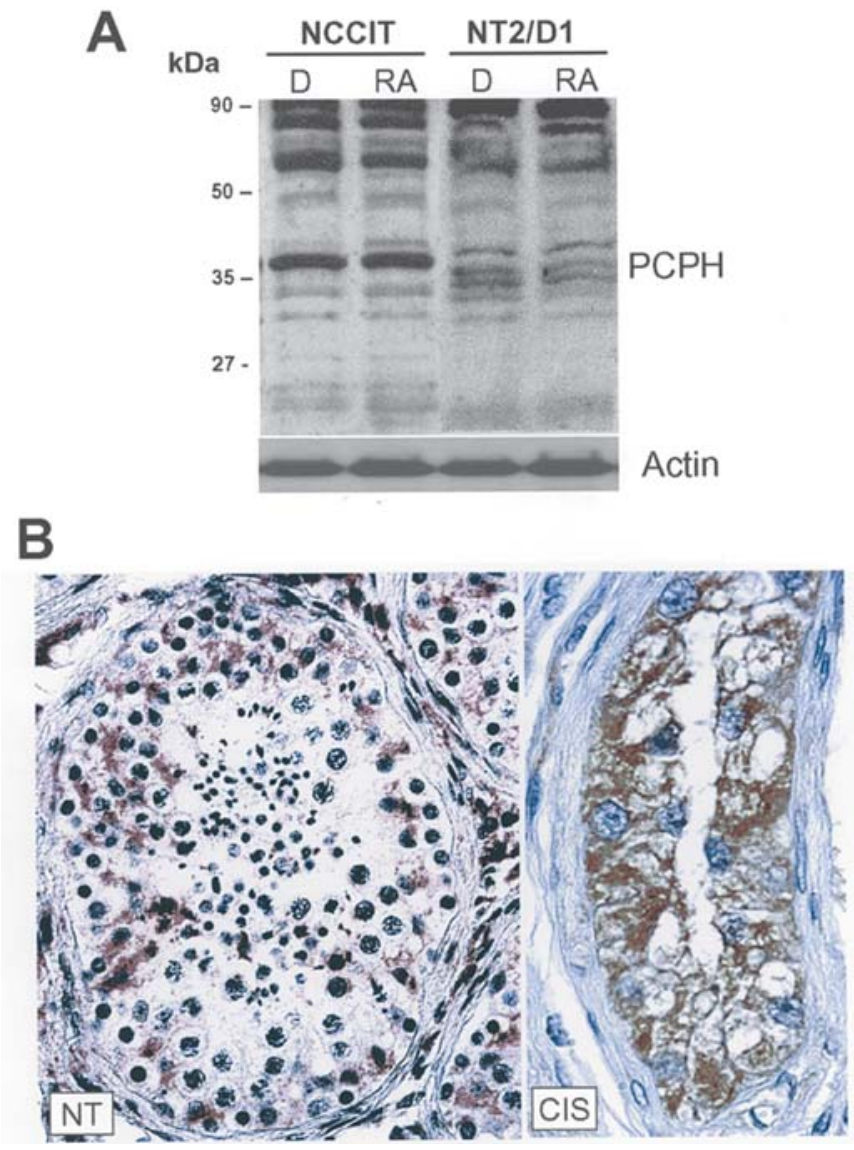

Figure 1. (A) Expression of PCPH in human TGCT cell lines. The media of exponentially growing replicated cultures of the cell lines indicated were supplemented with either a solution of all-trans retinoic acid (RA) in dimethyl sulfoxide to a final concentration of $10 \mu \mathrm{M}$, or with dimethyl sulfoxide alone (D). At different time intervals, the cells in individual culture replicas were collected and used to prepare total protein extracts, which were analyzed by Western immunoblotting with an anti-PCPH polyclonal antiserum (top panel) as described in the text. The blot shown corresponds to the samples collected after 2 weeks of RA addition. Western blots were stripped and re-probed using an anti-human B-actin antibody (lower panel) as a sample loading control. Molecular size markers are shown on the left. (B) Expression of PCPH in human testicular tissue. Normal testicle (NT) with complete spermatogenesis showing that $\mathrm{PCPH}$ staining is essentially undetectable in the basal spermatogonia, whereas low levels of staining are detectable in the adjacent Sertoli cells and in the Leydig cells. In contrast, samples of carcinoma in situ (CIS) showed an intense PCPH immunoreactivity in the cytoplasm of the tumor cells.

undetectable in germ line cells, including the spermatogonia (Fig. 1B, NT panel). On the contrary, PCPH expression was detected in neoplastic samples representative of various progression stages, including CIS (Fig. 1B, CIS panel). These results provided further impetus to investigate the expression of PCPH in a larger sample of TGCTs of different classes.

Immunohistochemical detection of PCPH in samples of seminoma and intratubular germ cell neoplasia (ITGCNU/CIS). Samples from 54 patients, representative of the major classes of TGCT, were analyzed for PCPH expression by immunohistochemical methods. Negative controls were performed on serial sections of the tumor samples by incubation without primary antibody. No background staining to $\mathrm{PCPH}$ was observed in any of these controls (data not shown). Samples from normal laryngeal mucosa were used as positive controls 


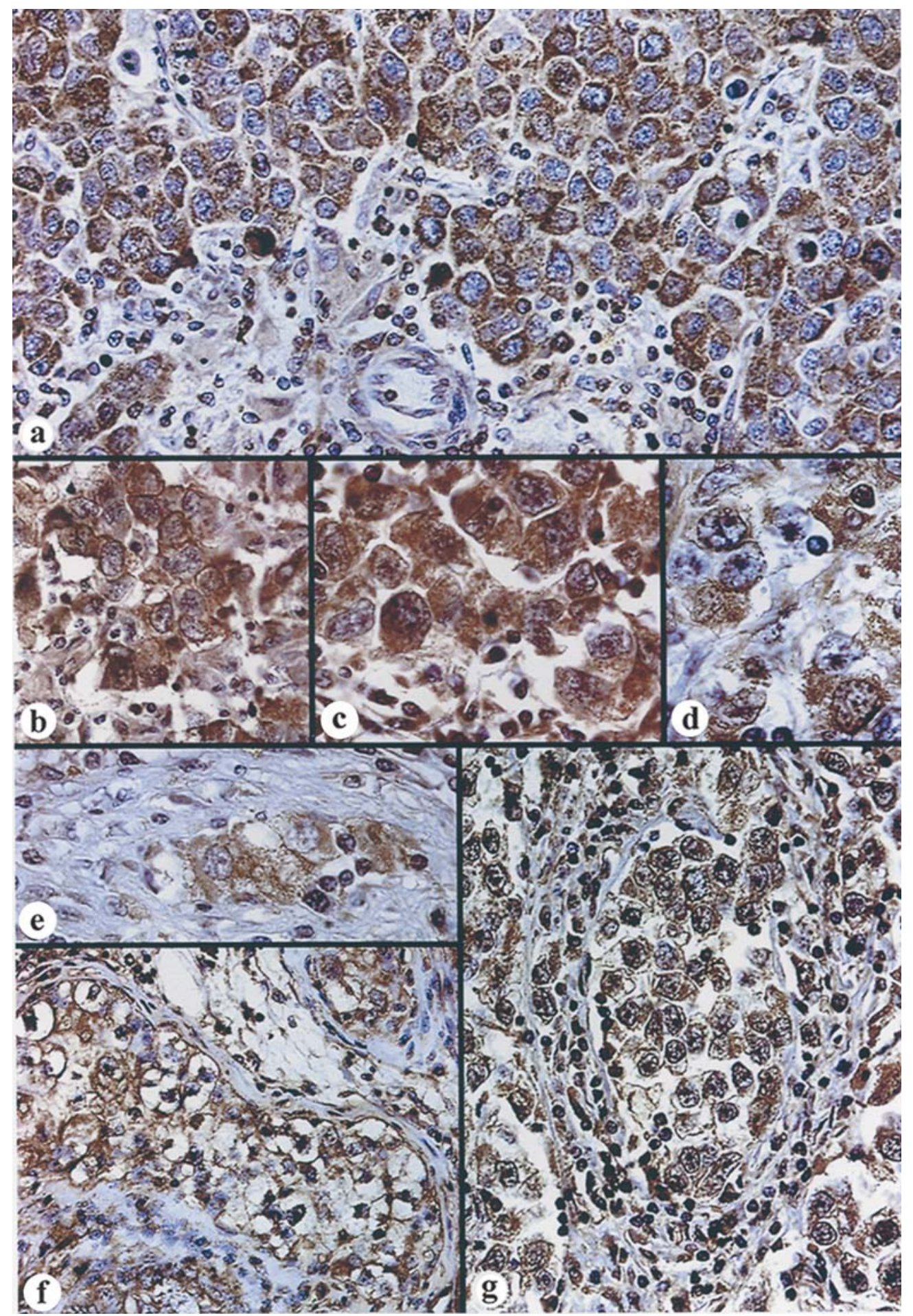

Figure 2. Expression of PCPH in samples of seminoma and testicular CIS. (a) Diffuse pattern of a seminoma showing intense PCPH expression in tumor cells while no staining in the interstitial connective tissue or in the peritumoral inflammatory cells, (b) honeycomb-like strong staining at the surface of the cells, (c) intense granular PCPH staining pattern in the cytoplasm, (d) moderate to light PCPH expression in the pleomorphic cells of a seminoma, (e) intense staining for PCPH in most of the cytoplasm in a carcinoma in situ (CIS) that developed in an atrophic seminiferous tubule, (f) seminiferous tubule filled by CIS cells, showing a characteristic vacuolation of their cytoplasm and the localization of PCPH staining towards the cellular periphery, (g) seminiferous tubule filled with CIS cells in an area of infiltrating seminoma, showing PCPH staining with similar intensities in CIS and seminoma cells. Cells were counterstained with hematoxylin in all cases. Magnification x40 (a, fand g) and x100 (b-e).

and yielded results consistent with previously published data (7). Analysis of the seminoma samples showed that PCPH was expressed in all cases. PCPH staining was granular and perinuclear in most seminoma cells (Fig. 2a), although in some cells staining was also observed in the cytoplasmic periphery (Fig. 2b and c). This staining pattern was the same regardless of the differentiation or pleomorphic characteristics of the cells (Fig. 2d). No staining for PCPH was detected in the lymphocytes present in the testicular seminoma samples. $\mathrm{PCPH}$ was also consistently expressed in all samples of carcinoma in situ (CIS/ITGCNU). Although the intensity of the PCPH staining was somewhat variable, CIS samples 


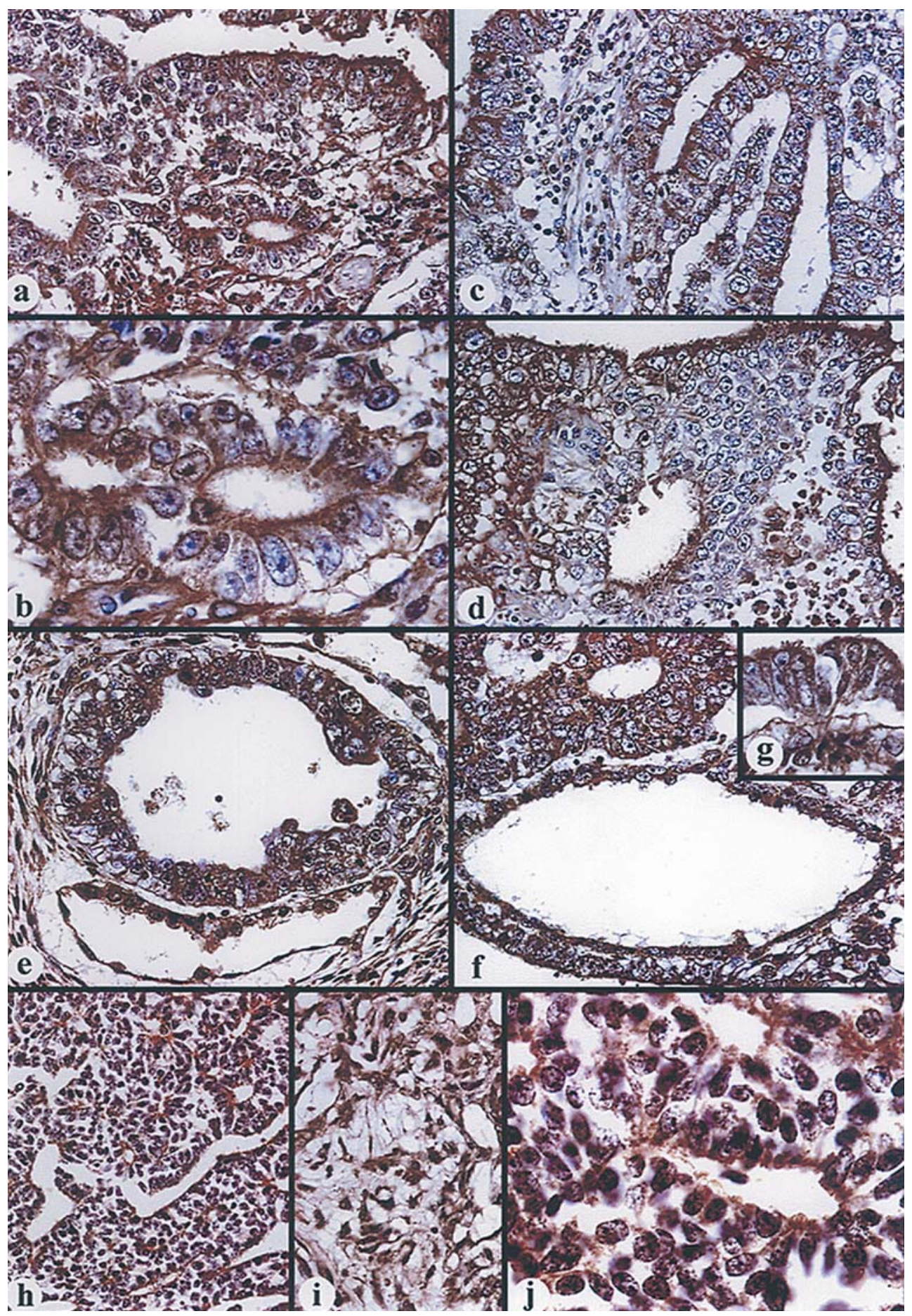

Figure 3. Expression of PCPH in samples of embryonal carcinoma and endodermal sinus tumors. (a) Embryonal carcinoma with glandular pattern showing intense PCPH staining in all tumor cells, (b) detail from the same sample demonstrating intense PCPH staining in the apical portion of the cytoplasm and in the villi projected from the cells, (c) variable expression of PCPH in an embryonal carcinoma combining glandular and solid patterns, (d) intense PCPH staining was detected in the glycocalix of the surface epithelium of glandular structures in embryonal carcinoma whereas only vestigial staining was detectable in adjacent dense areas, (e) embryoid body observed in a sample of embryonal carcinoma showing very intense staining for PCPH in the cells of the embryonal disk as well as in the cells lining the vitelline vesicle, (f) similar PCPH staining is observed in a more immature embryoid body, (g) embryoid body showing that PCPH staining is more in the cytoplasm and glycocalix of the epithelial cells in the embryoid disk than in the vitelline cells, (h) endodermal sinus tumors with combined solid and glandular patterns showing PCPH staining near the surface of cells lining the tumoral glands and crack-like structures, (i) endodermal sinus tumor with a mixoid solid pattern showing a light staining for PCPH in star-shaped cells, (j) moderate PCPH staining in the apical region of cells in an endodermal sinus tumor with glandular pattern whereas minimal staining was detectable in areas with solid proliferative pattern. Cells were counterstained with hematoxylin in all cases. Magnification x40 (a, c-f), x100 (b, g and j), and x10 (h and i).

frequently showed a granular pattern distributed throughout the cytoplasm (Fig. 2e). PCPH staining seemed to be displaced towards the cell periphery in cells with a high glycogen content (Fig. 2f), a characteristic of testicular CIS cells that results in their typically clear appearance. In cases in which CIS cells proliferate very actively, the peritubular wall stretches and the basal membrane may rupture, resulting in the conversion from CIS to an aggressive neoplasia that 
infiltrates the testicular interstitium. In these cases, all these malignant infiltrating cells expressed PCPH with the CIS characteristic pattern and intensity (Fig. 2g).

Immunohistochemical detection of PCPH in non-seminomatous tumors. Embryonal carcinoma may appear forming dense cellular packs as well as glandular structures, frequently showing ductal and papillary features. Typically, they consist of a mixture of areas showing these more defined patterns with others of marked proliferation (Fig. 3a-d), with a rather scarce stromal component and a lymphocyte population much smaller than in seminoma cases. In cases of embryonal carcinoma with a predominant glandular pattern (Fig. 3a and b), $\mathrm{PCPH}$ expression was located primarily around the nucleus, and there was an increase in $\mathrm{PCPH}$ expression towards the apical pole of their cytoplasm, being also positive in the villi projecting from these cells (Fig. $3 \mathrm{~b}$ and d). Additionally, $\mathrm{PCPH}$ was detected also in the lateral sides of the cytoplasmic membrane of embryonal carcinoma cells (Fig. 3c and d), near positions of intercellular adhesion structures. In contrast, only vestiges of perinuclear PCPH staining were detected in embryonal carcinoma samples showing a solid cellular pattern (Fig. 3d). PCPH was consistently expressed in the embryoid bodies present in most embryonal carcinomas (Fig. 3e and f), showing a double staining pattern in cytoplasmic granules and by the membrane, both in the cyclindrical epithelial cells of the embryonal carcinoma as well as in the cuboid and flat cells of the extraembryonal vesicles of the embryoid bodies (Fig. 3g).

PCPH was also consistently expressed in endodermal sinus tumors (yolk sac tumors) regardless of the tumors' architectural patterns: solid clusters with irregular cracks lined by tumor cells (Fig. 3h), reticular arrangements of anastomosed spindle-shaped cells with areas of mixoid degeneration (Fig. 3i), or glandular-like structures (Fig. 3j). The immunostaining intensity among endodermal sinus tumors was rather variable (Figs. 3h-3j) but always lighter than that observed in seminoma and in embryonal carcinoma samples with glandular patterns.

Immunohistochemical detection of $\mathrm{PCPH}$ in teratoma and mixed tumors. PCPH expression in teratoma samples was observed in tissue differentiated from the three primordial embryonal layers (Fig. 4). In cases of benign cystic teratoma (mature teratoma), the tissue derivatives of the three layers are intermingled in a disorganized manner, although organoid patterns are occasionally apparent. In both keratinized and non-keratinized squamous epithelia, PCPH showed a light pattern of granular expression in basal cells whereas, in squamous cells, $\mathrm{PCPH}$ immunoreactivity became preferentially perinuclear and increased in the cell periphery, resulting in a laminar staining with a honeycomb-like characteristic pattern (Fig. 4a and b). This staining pattern was progressively lost in the most superficial cells of the squamous stratum and completely disappeared in the stratum corneum.

$\mathrm{PCPH}$ expression was quite high in endodermal derivatives, especially in glandular structures (Fig. 4c) as well as in intestinal and airway surface epithelia (Fig. 4d and e). PCPH staining was very strong in the enterocytic glycocalix, but no staining was observed in the large mucinous vacuoles of goblet cells (Fig. 4d). In other areas, mature teratomas include cuboid less-differentiated surface epithelia in which PCPH immunoreactivity always appeared to be less intense than in welldifferentiated epithelia (Fig. 4e). In transitional epithelia, including the urothelium, PCPH showed a peculiar distribution: a membrane associated pattern, with a marked and strong staining in the most superficial portion of the urothelial cytoplasm (Fig. 4f). On the other hand, the cytoplasm of the smooth muscle cells in the wall of the intestinal endodermal derivatives showed more intense $\mathrm{PCPH}$ staining than that detected in fibroblasts of the tumoral stroma (Fig. 4d).

Immature teratomas often include areas with solid cellular arrangements, in which immature neuroepithelial derivatives predominate, intermingled with irregular cystic formations. In addition, the proliferative mesenchyme is rather disorganized, shows a high cellular density and frequent mitoses. In these samples (Fig. 4g and h), PCPH expression was detected in ecto-endodermal derivatives as well as in neuroepithelial and mesodermal derivatives, although the expression level in the latter types was consistently lighter than in the adjacent epithelial derivatives (Fig. 4h). PCPH immunoreactivity was also rather light in areas containing fibroblast-like, very immature mesenchymal cells and highly pleomorphic smooth muscle cells with obvious signs of dedifferentiation (Fig. 4i), which could be appreciated in some cases that resembled leiomyosarcomas.

Testicular tumors of combined/mixed pathologies proved to be good models for the study of PCPH expression, because they recapitulated the peculiarities of PCPH expression in each of the single pathologies contributing to the mixed nature of the tumor samples evaluated. Results from the immunohistochemical analysis of mixed TGCT samples confirmed that PCPH expression levels were generally higher in well differentiated tumor cells, both in the mature epithelia of the teratomatous components as well as in the adjacent areas of embryonal carcinoma (Fig. 4j), and that there was a progressive loss of $\mathrm{PCPH}$ staining in cases showing changes in the maturation or dedifferentiation of surface epithelia of benign teratomatous cysts and of the adjacent areas of embryonal carcinoma within the same tumors (Fig. 4k).

Differential expression of PCPH mRNA and polypeptides between normal testicular tissue and TGCTs. Whenever possible, given the extent of tumoral spread and infiltration, matched samples of macroscopically normal tissue and tumors were collected from the same patient. Total tissue extracts from these matched samples were prepared to isolate mRNA for RT-PCR analyses or to obtain total protein preparations for Western immunoblot assays. Representative results from these experiments are shown in Fig. 5. Consistent with immunohistochemical data, a 1448-bp RT-PCR $P C P H$ fragment was detectable in the majority of the samples for which frozen matched normal tissue and tumor materials were available, while it remained undetectable in a small number of the samples tested (Fig. 5A). Although the levels of mRNA expression were generally low, relative to those in the positive control HT-29 cells, and varied significantly among normal tissue from different patients, results clearly showed that $P C P H$ expression was markedly increased in TGCTs relative to their matched normal counterparts (Fig. 5A). Western 


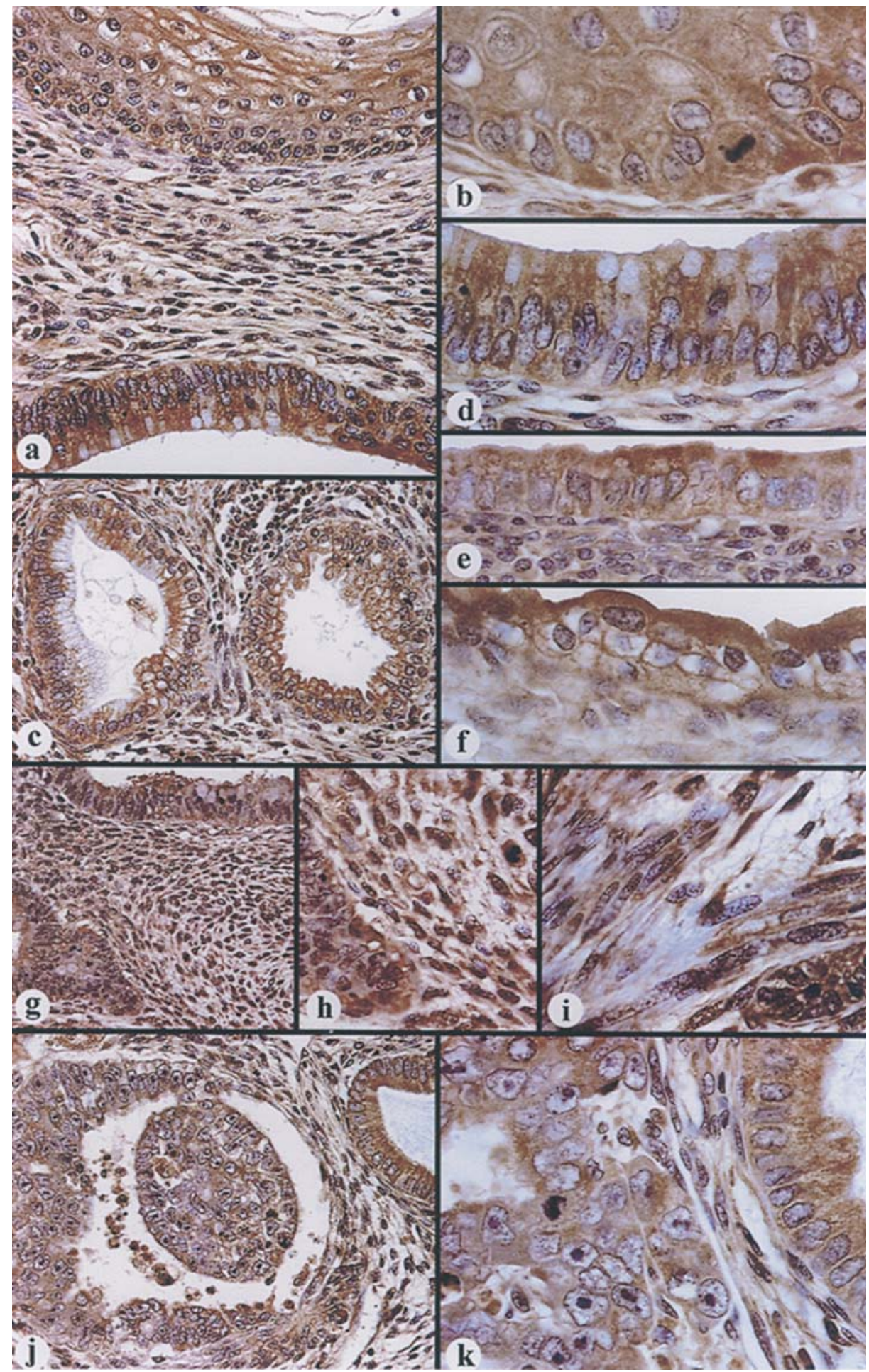

Figure 4. Expression of PCPH in samples of teratoma and mixed TGCTs with teratomatous components. (a) Mature teratoma with multiple cysts showing an upper portion differentiated into a keratinized, stratified epithelium with PCPH staining in a typical honeycomb pattern, a lower portion showing a cylindrical surface epithelium with strong PCPH staining, and the mesenchyme with some spindle-shaped cells showing positive, but less intense, staining for PCPH; (b) basal and parabasal cells of the squamous epithelium with very intense PCPH staining, which remains in the mitotic cell shown; (c) glandular structures with strong PCPH staining: the left gland shows mucous intestinal cells with intense staining at the basal cytoplasm and no staining in the mucinous glands, and the right gland showing intense staining in all apocrine epithelial cells; (d) enterocytes intensely labeled for PCPH and goblet cells with moderate expression localized mainly in the basal cytoplasm and perinuclearly, while some smooth muscle cells contain PCPH in a fibrilar arrangement; (e) moderately differentiated cuboid surface epithelium clearly showing less intense staining for PCPH, with lymphocytes in the lamina propia being negative for PCPH staining; (f) urothelial-like differentiation with intense PCPH staining around the cuticle of the superficial zone of the epithelial cells; (g) immature teratoma showing intense PCPH staining in the well-differentiated surface epithelium and lower staining in the poorly differentiated adjacent mesenchyme; (h) detail from the previous panel showing that, although immature mesenchymal cells stain lightly for PCPH, those undergoing mitosis clearly express PCPH; (i) pleomorphic smooth muscle cells in an immature teratoma show very little or no staining for PCPH; (j) mixed TGCT with intense PCPH staining in the welldifferentiated epithelium that line the teratoma cavity and constituting the embryonal carcinoma; $(\mathrm{k})$ mixed TGCT with moderate differentiation of the glandular epithelium of the benign teratomatous cyst associated with the malignant epithelium of the embryonal carcinoma, but both showing lower PCPH staining than the sample shown in the previous panel. Cells were counterstained with hematoxylin in all cases. Magnification x40 (a, c, g and j) and x100 (b, d-f, h, I and k). 
A

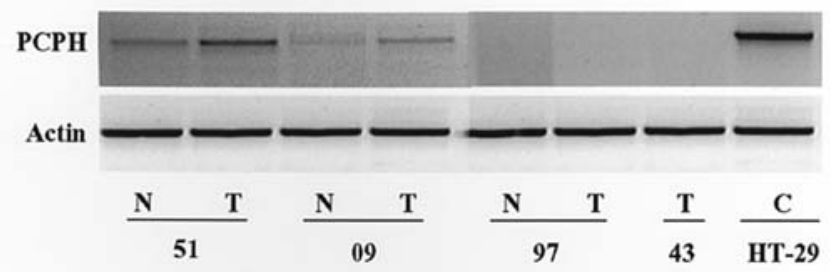

B

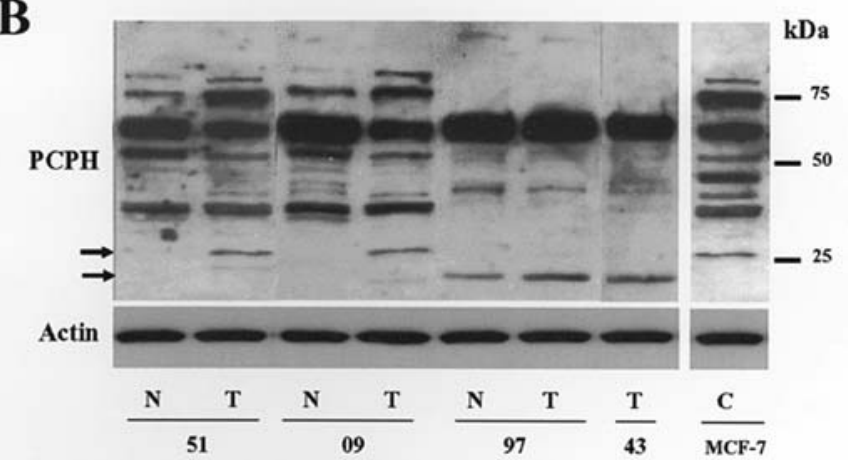

Figure 5. Differential expression of PCPH in matched normal (N) and tumor (T) samples from the same patients. Representative data from (A) RT-PCR analysis of $P C P H$ mRNA expression levels and (B) Western immunoblot analysis of the same selected samples. Human colon carcinoma HT-29 (A) and breast carcinoma MCF-7 cells (B) were used as positive controls for mRNA and protein analyses, respectively. Increased expression levels of $P C P H$ mRNA in tumor tissue correlated with the expression in the same samples of low molecular mass polypeptides (arrows in B) that were undetectable in the matched normal tissue.

immunoblot analyses of the same paired samples showed that PCPH-immunorelated polypeptides were detected in all of the samples tested, even in those that yielded negative results in RT-PCR. The pattern of PCPH polypeptides was very similar to that previously described for TGCT cell lines (Fig. 1A) as well as to the well characterized pattern of the MCF-7 human breast carcinoma (5), which was used as a positive control (Fig. 5B). Comparison between the polypeptide patterns in TGCTs and their normal controls showed that the most remarkable differences corresponded to low molecular mass PCPH polypeptide species. The majority of TGCT cases expressed a polypeptide of approximately $27 \mathrm{kDa}$ that was absent in their normal controls (Fig. 5B, samples \#51 and $\# 09$, which also had increased levels of $P C P H$ mRNA). In other cases, neither normal tissue nor TGCT samples expressed the $27 \mathrm{kDa}$, but tumors showed increased expression of another low molecular mass PCPH polypeptide of approximately $20 \mathrm{kDa}$ (Fig. 5B, samples \#97 and \#43).

\section{Discussion}

Results from the study described here represent the first report on the possible involvement of the product of the $\mathrm{PCPH}$ gene in the development of TGCT. Our data conclusively show that PCPH is expressed in human TGCT cell lines (Fig. 1A) and tumors (Figs. 2-5). The fact that the tested cell lines differ in the status of their Tp53 tumor suppressor protein suggests that PCPH expression is independent of the functionality of Tp53, although PCPH has been reported to interact with the Tp53-mediated DNA damage response in certain cell types (4).
With regard to the expression in neoplastic samples, the marked expression of PCPH in the cytoplasm of cells of ITGCNU/CIS cases (Figs. 1B and 2e-2g) and the absence of PCPH expression in germ line cells, including the spermatogonia, of normal subjects (Fig. 1B, NT panel) suggests that PCPH may play a carcinogenic role in TGCT progression. Furthermore, the consistent immunohistochemical detection in samples of ITGCNU/CIS, the recognized TGCT precursor lesion (13), of levels of PCPH expression greater than those in normal tissue (Fig. 1B) strongly indicates that molecular alterations leading to $\mathrm{PCPH}$ deregulation may be involved in early stages of TGCT development. This is important because, although TGCTs are highly curable malignancies $(21,24)$ and recent technological advances have allowed the characterization of global alterations in gene expression in TGCTs (32-36), identification of individual molecular markers at the earliest tumor stages may be particularly useful to minimize therapeutic intervention. Our results showing the increased expression of PCPH in testicular tumors relative to their matched normal counterparts (Fig. 5) are also consistent with reports of chromosomal abnormalities, most frequently gains, in mouse and human testicular tumors involving the syntenic regions of mouse chromosomes 12 and human chromosome 14 (14q13.3-14qter), where the $P C P H$ gene was previously mapped to $(26,27)$.

Our results show that $\mathrm{PCPH}$ is present in well-differentiated squamous epithelia, while it seems to be progressively lost in dedifferentiated squamous cells (Figs. 2-4), a pattern similar to that described previously for PCPH in samples of human laryngeal dysplasia (7), in which PCPH is expressed at lower levels in severe than in mild dysplastic cases and at much lower levels than in the normal laryngeal mucosa (7). The preferential expression of PCPH in well-differentiated slowly proliferating cells rather than in less-differentiated more actively proliferating cells is consistent with the detection in mature teratoma samples of higher levels of PCPH expression in cells of the basal layer than in suprabasal cells (Fig. 4a and b), which function to renew the epithelium and thus have a greater proliferative capacity. Another interesting observation was that, whereas dense areas of embryonal carcinoma containing dedifferentiated cells (Fig. 3d) express low levels of PCPH located only in the perinuclear compartment, $\mathrm{PCPH}$ is primarily located in the lateral sides of the cylindrical cells of embryonal carcinoma samples with glandular pattern (Fig. 3c and d). The fact that a similar location has been observed in normal squamous epithelia (larynx and skin) (7), in dermoid cysts of the ovarian squamous epithelium (unpublished observation) and in samples of well-differentiated teratoma (Fig. 4), may suggest a common function of $\mathrm{PCPH}$ perhaps related to mechanisms regulating cell adhesion and/or cell-cell interaction. In addition, the presence of PCPH at the cell surface in the apical pole and the villi (Fig. $3 b$ and d) in glandular embryonal carcinoma samples may suggest that PCPH might be secreted to the extracellular compartment by the neoplastic cells. The presence of a putative signal peptide in the primary sequence of PCPH, deduced by translation of its nucleotide sequence $(2,26,27)$, may satisfy the necessary structural/ functional requirements to allow for PCPH secretion.

It is noteworthy that even normal testicular tissue expresses multiple PCPH polypeptide forms (Fig. 5B) with molecular 
masses of approximately 20 to $>90 \mathrm{kDa}$. To date, this is the first case of a normal tissue simultaneously expressing the various PCPH forms that are normally expressed as individual polypeptides in other tissues and organs $(6,26,27)$, a phenomenon most likely caused by alternative promoter usage and/or splicing abnormalities (unpublished data) and only observed previously at various stages of development of other rodent and human tumor types (6-8). However, the existence of multiple PCPH forms in normal testicular tissue is consistent with the known high levels of alternative splicing in testes $(37,38)$ and with the fact that alternative $P C P H$ transcripts could be detected in mRNA preparations from normal testes of all the species tested thus far (25). The apparent lack of correlation between RT-PCR data indicating the absence of $P C P H$ transcripts in certain normal and tumor samples (Fig. 5A) and the results from Western analyses demonstrating the expression of $\mathrm{PCPH}$ polypeptides in the same samples may be due to the possible differences in either transcript or polypeptide stability in those samples or to the loss of sequences needed for the annealing of one or both of the RT-PCR primers caused by alternative splicing mechanisms.

The mechanism of oncogenic activation of the $P C P H$ gene is known to involve the deletion of a single base pair within its open reading frame (ORF) (2). This point-mutational event shifts the normal ORF, causing the translation of the mutated protein to terminate 33 amino acid residues downstream of the mutated codon. Consequently, the PCPH oncoprotein is a truncated form of the normal gene product, contains an additional hydrophobic C-terminal tail, and has a molecular mass of approximately $27 \mathrm{kDa}$ (2). Taking into consideration that the $27-\mathrm{kDa} \mathrm{PCPH}$ polypeptide detected in TGCT samples showed an apparent molecular mass similar to that of the PCPH oncoprotein and that it was absent from the matched normal tissue, it seems possible that the $27-\mathrm{kDa}$ polypeptide may be the product of an activated $P C P H$ oncogene present in testicular tumors. Unfortunately, direct detection of a possibly similar mutational event by sequencing techniques was not feasible due to the limited sample amounts available. However, although it remains to be conclusively demonstrated as to whether the $27-\mathrm{kDa} \mathrm{PCPH}$ polypeptide detected in TGCT truly reflects the expression of a PCPH oncoprotein, the likelihood of this being the case has recently received strong support from data obtained in our laboratory from experiments to determine whether post-translational modifications unique to, or lost by, the PCPH oncoprotein as a consequence of the truncating mutational event described above (2) were also gained or lost by the $27-\mathrm{kDa}$ polypeptide immunoprecipitated from total extracts of human cell lines derived from tumors of different types. The results (data not shown) consistently revealed similar phosphorylation signatures at serine and threonine residues, and confirmed the expected losses of arginine dimethylation and the phosphorylation at a unique tyrosine residue.

Overall, our results identify $\mathrm{PCPH}$ as a good molecular marker for TGCTs. Furthermore, the detection in TGCTs of small PCPH polypeptides that are absent in matched normal tissue from the same patients strongly indicates that immunodetection of PCPH polypeptides may be a useful tool for early diagnosis of testicular neoplasias.

\section{Acknowledgments}

This work was supported by USPHS grant RO1-64472 from the National Cancer Institute. Partial support was also received through grant PI021188 from the Fondo de Investigaciones Sanitarias of the Spanish Ministry of Health.

\section{References}

1. Velasco JA, Castro R, Avila MA, Laborda J, Di Paolo JA, Cansado J and Notario V: $c p h$, a novel oncogene which cooperates with H-ras in the transformation of NIH/3T3 fibroblasts. Oncogene 9: 2065-2069, 1994.

2. Velasco JA, Avila MA and Notario V: The product of the $c p h$ oncogene is a truncated, nucleotide binding protein that enhances cellular survival to stress. Oncogene 18: 689-701, 1999.

3. Recio JA, Páez JG, Sanders S, Kawakami T and Notario V: Partial depletion of intracellular ATP mediates the stress-survival function of the PCPH oncoprotein. Cancer Res 62: 2690-2694, 2002.

4. Tirado OM, Mateo-Lozano S, Sanders S, Dettin LE and Notario V: The PCPH oncoprotein antagonizes the pro-apoptotic role of mTOR in the response of normal fibroblasts to ionizing radiation. Cancer Res 63: 6290-6298, 2003.

5. Rouzaut A, Recio A and Notario V: Expression of the protein product of the PCPH proto-oncogene in human tumor cell lines. Radiat Res 155: 181-187, 2001.

6. Solanas M, Escrich E, Rouzaut A, Costa I, Martínez A and Notario V: Deregulated expression of the $P C P H$ proto-oncogene in rat mammary tumors induced with 7,12-dimethylbenz $(a)$ anthracene. Mol Carcinog 33: 219-227, 2002.

7. Blánquez MJ, Regadera J, Mariño J, Newman RE and Notario V: Gradual deregulation and loss of PCPH expression in the progression of human laryngeal neoplasia. Mol Carcinog 35: 186-195, 2002.

8. Blánquez MJ, Arenas MI, Conde I, Tirado OM, Paniagua R and Notario V: Deregulated expression of the $P C P H$ proto-oncogene in human breast cancers. Int J Oncol 25: 821-830, 2004.

9. Lifschitz-Mercer B, Elliott DJ, Schreiber-Bramante L, LeiderTrejo L, Eisenthal A and Bar-Shira Maymon B: Intratubular germ cell neoplasia: associated infertility and review of the diagnostic modalities. Int J Surg Pathol 9: 93-98, 2001.

10. Oosterhuis JW and Looijenga LHJ: Testicular germ-cell tumours in a broader perspective. Nat Rev Cancer 5: 210-222, 2005.

11. Reuter VE: Origins and molecular biology of testicular germ cell tumors. Mod Pathol 18: S651-S660, 2005.

12. Ulbright TM: Germ cell tumors of the gonads: a selective review emphasizing problems in differential diagnosis, newly appreciated, and controversial issues. Mod Pathol 18: S61-S79, 2005.

13. Rajpert-De Meyts E, Bartkova J, Samson M, Hoei-Hansen CE, Frydelund-Larsen L, Bartek J and Skakkebaek NE: The emerging phenotype of the testicular carcinoma in situ germ cell. APMIS 111: 267-279, 2003 .

14. Bosl JG and Motzer RJ: Testicular germ-cell cancer. N Engl J Med 337: 242-253, 1997.

15. Foster RS: Early-stage testis cancer. Curr Treat Options Oncol 2: 413-419, 2001

16. Garzotto $M$ and Nichols CR: Current concepts in risk factor assessment for advanced germ cell cancer. Semin Urol Oncol 19: 165-169, 2001.

17. Mickisch GH: Prognostic parameters for the management of advanced testis tumours. Curr Opin Urol 10: 465-471, 2000.

18. Burger H, Nooter K, Boersma AWM, Kortland CJ, van den Berg AP and Stoter G: Expression of P53, P21/WAF/CIP, BCL-2, BAX, BCL-X, and BAK in radiation-induced apoptosis in testicular germ cell tumor lines. Int J Radiat Oncol Biol Phys 41: 415-424, 1998.

19. Burger H, Nooter K, Boersma AWM, van Wingerden KE, Looijenga LHJ, Jochemsen AG and Stoter G: Distinct p53independent apoptotic cell death signalling pathways in testicular germ cell tumor cell lines. Int J Cancer 81: 620-628, 1999.

20. Takayama H, Takakuwa T, Tsujimoto Y, Tani Y, Nonomura N, Okuyama A, Nagata S and Aozasa K: Frequent Fas gene mutations in testicular germ cell tumors. Am J Pathol 161: 635-641, 2002. 
21. Spierings DCJ, De Vries EGE, Vellenga E and De Jong S: The attractive Achiles heel of germ cell tumours: an inherent sensitivity to apoptosis-inducing stimuli. J Pathol 200: 137-148, 2003.

22. Richards EH, Hickey E, Weber L and Master JR: Effect of overexpression of the small heat shock protein HSP27 on the heat and drug sensitivities of human testis tumor cells. Cancer Res 56: 2446-2451, 1996.

23. Shenck PW, Stoop H, Bokemeyer C, Mayer F, Stoter G, Oosterhuis JW, Wiemer E, Looijenga LHJ and Nooter K: Resistance to platinum-containing chemotherapy in testicular germ cell tumors is associated with downregulation of the protein kinase SRPK1. Neoplasia 6: 297-301, 2004.

24. Einhorn LH: Chemotherapeutic and survival strategies for germ cell tumors. Chest Surg Clin North Am 12: 695-706, 2002.

25. Velasco JA, Zimonjic DB, Popescu NC, Cansado J, Di Paolo JA, Albor A and Notario V: Tissue-specific expression, evolutionary conservation and localization of the cph proto-oncogene on Syrian hamster chromosome X. Oncogene 12: 2713-2717, 1996.

26. Recio JA, Zambrano N, De la Peña L, Powers C, Siwarski D, Huppi K and Notario V: cDNA isolation, expression and chromosomal localization of the mouse $P c p h$ proto-oncogene. Mol Carcinog 26: 130-136, 1999.

27. Recio JA, Zambrano N, De la Peña L, Reig JA, Rhoads A, Rouzaut A and Notario V: The human $P C P H$ proto-oncogene: cDNA identification, primary structure, chromosomal mapping and expression of in normal and tumor cells. Mol Carcinog 27: 229-236, 2000

28. Coenen Schimke JM, Ljungqvist OH, Sarkar G, Conover CA and Sreekumaran Nair K: A quantitative PCR measurement of messenger RNA expression of IGF-I, IGF-II and IGFBP-5 in human skeletal muscle. Growth Horm IGF Res 9: 179-186, 1999.

29. Wang JA, Fan S, Yuan RQ, Ma YX, Meng Q, Goldberg ID and Rosen EM: Ultraviolet radiation down-regulates expression of the cell-cycle inhibitor p21WAF1/CIP1 in human cancer cells independently of p53. Int J Radiat Biol 75: 301-316, 1999.
30. Damjanov I, Horvat B and Gibas Z: Retinoic acid-induced differentiation of the developmentally pluripotent human germ cell tumor-derived cell line, NCCIT. Lab Invest 68: 220-232, 1993.

31. Spinella MJ, Kitareewan S, Mellado B, Sekula D, Khoo KS and Dmitrovsky E: Specific retinoid receptors cooperate to signal growth suppression and maturation of human embryonal carcinoma cells. Oncogene 16: 3471-3480, 1998.

32. Kelland LR, Bingle L, Edwards S and Steel GG: High intrinsic radiosensitivity of a newly established and characterized human embryonal rhabdomyosarcoma cell line. Br J Cancer 59: 160-164, 1989.

33. Von Eyben FE: Chromosomes, genes, and development of testicular germ cell tumors. Cancer Genet Cytogenet 151: 93-138, 2004

34. Hoei-Hansen CE, Nielsen JE, Almstrup K, Hansen MA, Skakkebaek NE, Rajoert-De Meyts E and Leffers H: Identification of genes differentially expressed in testes containing carcinoma in situ. Mol Hum Reprod 10: 423-431, 2004.

35. McIntyre A, Summersgill B, Jafer O, Rodriguez S, Zafarana G, Oosterhuis JW, Gillis AJM, Looijenga L, Cooper C, Huddart R, Clark J and Shipley J: Defining minimum genomic regions of imbalance involved in testicular germ cell tumors of adolescents and adults through genome wide microarray analysis of cDNA clones. Oncogene 23: 9142-9147, 2004.

36. Korkola JE, Houldsworth J, Dobrzynski D, Olshen AB, Reuter VE, Bosl GJ and Chaganti RS: Gene expression-based classification of nonseminomatous male germ cell tumors. Oncogene 24: 5101-5107, 2005.

37. Venables JP: Alternative splicing in the testes. Curr Opin Genet Dev 12: 615-619, 2002.

38. Yeo G, Holte D, Kreiman G and Burge CB: Variations in alternative splicing across human tissues. Genome Biol 5: R74, 2004. 